\title{
Der Infektabwehrmechanismus der Harnblase
}

Es ist bekannt, dass Frauen häufiger als Manner an Harnwegsinfektio-nen leiden. Für diese Tatsache werden gewöhnlich anatomische Ursachen verantwortlich gemacht. Es ist wahrscheinlich, dass die Krankheitserreger aus dem Patientendarm über die Harnröhre in die Blase und aufsteigend in die Nierenbecken gelangen. Die Tatsache der kürzeren weiblichen Urethra bietet allerdings allein keine ausreichende Erklärung für die unter-schiedliche Infektionsanfälligkeit, nachdem Hinman nachweisen konnte, dass in der Harnröhre der Frau unter Turbulenzerscheinungen periphere Anteile des Urinstromes umgeleitet werden und mit ihm Krankheitserreger rückläufig in die Blase gelangen. Wenn angenommen wird, dass es sich hierbei um einen häufig zu beobachtenden Vorgang handelt, müssen Me-chanismen bestehen, die eine Vermehrung der eingedrungenen Keime im Blasenurin verhindern. Eine Erklärung mit Hilfe der bisherigen Erkennt-nisse ist nicht möglich, da die Einfüllung eines stark bakterizid wirkenden Mittels in die leere Blase notwendig ist, um den grössten Teil der in die Blase eingedrungenen Erreger nach der Miktion zu vernichten.

Ein solches Material konnte jetzt von Stamey et al. im Prostatasekret nachgewiesen werden. Unter Pilocarpinstimulierung gelang es bei Hunden, bis zu $200 \mathrm{ml}$ Prostatasekret zu gewinnen, das in der Lage war, einen grossen Teil aller bekannten Erreger von Harnwegsinfektionen sofort $\mathrm{zu}$ vernichten. An Patienten konnten sie ferner nachweisen, dass im Mittelstrahlurin Bakterienwachstum auftrat, während dies bei kleinen Harnportionen nach Bla-senentleerung und Prostatamassage nicht der Fall war.

Dieser antibakterielle Effekt wird offensichtlich von einem hitzestabi-len Stoff mit niedrigem Molekulargewicht hervorgerufen, der nicht aus dem Serum stammt und der sich hinsichtlich seines antibakteriellen Spek-trums sowie anderer Eigenschaften von Sperminen und Lysozymen unter-scheidet.

Eine solche, nach der Miktion antibakteriell wirkende Substanz stellt gemeinsam mit der Urinverdünnung durch Harnzufluss nach einer Blasen-entleerung einen soliden Abwehrmechanismus gegen Infektionen dar.

1 Originaltitel: Defence mechanisms of the bladder.

\section{SCHÄFER}

109

Diese Beobachtung, die allerdings noch viele Fragen offen lässt, wie zum Beispiel die der Ursache des Geschlechtsunterschiedes, wird zum Ver-ständnis der Abwehrvorgänge bei Harnwegsinfektionen beitragen.

V. Schäfer

Aus: Lancet, No. 7552: 1112-1114 (1968); cit. Gynäk. Rdsch. 7: 109-110 (1969)

Suprapubische Aspiration yon Urin während der Schwang $\beta r-$ schaEt1

I. R. McFadyen und S. J. Eykyn

St. Thomas' Hospital, Medical School, London (England) 
Die Verfasser fahndeten bei 1000 Schwangeren nach einer Harnwegs-infektion, wobei sie zur Gewinnung des Urins die perkutane suprapubische Aspiration (S. P. A.) benutzten.

Komplikationen wurden bei der Bla-senpunktion nicht beobachtet. Bei 5,9\% der Graviden fand sich eine Bakteriurie (60\% E. coli; 13,9\% Paracoli; 9,2\% Proteus; 10,8\% Staphy-lococcen; 6,1 \% andere Erreger). Dieser Feststellung kommt besondere Bedeutung zu, da jede Kontamination des Harns mit Urethra und Vagina vermieden werden konnte. Die Bakteriurie blieb bestehen, wenn eine ent-sprechende Behandlung unterlassen wurde, in $57 \%$ entwickelten sich dann klinische Symptome. Bemerkenswert ist, dass bei 15,9 \% aller Frauen Hämophilus vaginalis im Urin nachgewiesen wurde.

Zur Gewinnung von Urin für eine exakte bakteriologische Untersu-chung und Auswertung betrachten die Verfasser die Methode der S.P.A. als besonders geeignet, wobei darauf hingewiesen wird, dass $98 \%$ aller Frauen mit einem sterilen Blasenurin keinen bakterienfreien Mittelstrahl-urin produzieren können, auch wenn optimale Vorbereitungen zur Gewinnung getroffen werden. Bei der Entnahme von Katheterurin verweisen sie auf die Gefahr der Keimverschleppung in die Blase und einige andere Nachteile dieses Vorgehens. 1 Originaltitel: Suprapubic aspiration of urine in pregnancy. 